reviewers and reduce the time required to conduct SRs is automation.

With recent advancements in artificial intelligence and machine learning, many tools have been, and are currently being, developed to support different stages of SR process. To date, the range of automated tools available, and their effectiveness, is unclear. To remedy this gap, we have undertaken a methodological systematic review.

The specific objectives of this methodological SR are to:

- Classify existing tools according to the automation approach, the stage of SR supported and the level of automation.

- Compare the available tools according to each stage that they support and identify their strengths and limitations.

- Determine the effectiveness of the tools.

- Present and appraise evaluations of automated tools.

Method This review is based on the Preferred Reporting Items for Systematic review and Meta-Analyses (PRISMA-P) Guidelines.

We reviewed all published articles, grey literature, reports and software manuals that evaluate automated and semi-automated tools that support healthcare-related SRs, from screening to write-up. Only tools that are fully developed were eligible for inclusion.

The systematic review toolbox (SR ToolBox), PubMed, Google and Google were systematically searched. Titles, abstracts and full articles were reviewed for inclusion independently by two reviewers. Data extraction and quality appraisal were undertaken independently by two reviewers, with disagreements resolved by consensus or by arbitration by a third reviewer if necessary.

Tools' characteristics and performance metrics reported in the included studies were extracted and tabulated. To enable comparisons, tools were grouped according to stages of SR they support, and the type of algorithms deployed in them.

Results This review is currently in the data extraction stage and will be completed by the first week of June 2019 .

This presentation will focus on the evidence available on automated tools that support the screening, data extraction, quality appraisal and write-up phases of SRs. For each phase, we will present data on the number of tools that have been developed and the number of studies that have evaluated them. We will discuss the strengths and limitations of the methods and standards used to evaluate existing tools, and implications for future research and SR practice.

Conclusions This review constitutes an important step in easing the transition of SR production from a primarily manual process to a semi-automated one. It will inform current collaborative efforts aimed at the development of evidenceinformed integrative automated systems for conducting high quality SRs in healthcare research.

\section{SUSTAINING AND DEVELOPING MOVEMENTS, LIKE EVIDENCE LIVE, IN MEDICINE: LESSONS TO BE LEARNT WHEN DOES A CAMPAIGN BECOME A MOVEMENT - AND HOW DO WE IMPLEMENT A MOVEMENT INTO PRACTICE?}

\footnotetext{
${ }^{1,2}$ Neil Chanchlani. ${ }^{1}$ Royal Devon and Exeter Hospital, Exeter, UK; ${ }^{2}$ Canadian Medical Association, Ottawa, Canada
}

Social movements across medicine, research, and publishing, such as EBM Live, Choosing Wisely, and Reducing Research Waste are well-intentioned, but are met with varying degrees of success. We need to learn from recent successful social movements that have reached international circulation and had fundamental impact, such as \#MeToo and Black Lives Matter. The EBM manifesto's aim to reduce bias, wastage, error fraud research is driven by a small community of researchers, academics, and publishers, largely from the United Kingdom. What percentage of end users, including researchers, patients, and public, are engaged or even aware of the movement? We must move away from simply raising awareness amongst small communities to co-producing and collaborating with them. Looking at successful social movements within and outside medicine that have generated transformational change and inspired the public, the following themes emerge:

1. Have a clear purpose and vision for the movement. This has to be simple, short, and direct rather than a complicated and detailed plan. This will hook and unify the targeted audience and break down interdisciplinary barriers. (Satell, Harvard Business Review 2016).

2. Use a structured framework to ensure standardisation across the movement. In order to enact change locally, nationally, and internationally, there needs to be clear guidance. For example, the Knowledge-to-Action Cycle, generated by Canadian Institute of Health Research, can be used as a guiding framework to increase the relevance, applicability, and impact of research findings (Field, Implementation Science 2014). This will increase clarity and process within the movement.

3. Training nominated champions will increase uptake of the movement. Nominated champions should undergo sponsored training which will empower them to create a shared vision amongst their teams within hospital, university, or organisation. Formal accreditation, such as the Knowledge Translation Professional Certificate ${ }^{\mathrm{TM}}$, or informal opportunities, such as workshops and summer courses run by Evidence Live are a starting point. Cementing awareness can be formalised through the National Institute for Health Research Good Clinical Practice module and local induction for all healthcare and university staff, and not just those involved in research. This will ensure everyone is involved and engaged in the movement.

4. Continuous monitoring and evaluation are necessary for a successful movement. An iterative process will allow strategy review, monitoring of activities, and amendments to be made in line with the movement's values. Sustaining a movement in medicine is difficult. For real change to occur, the whole community needs to be engaged. Evidence Live is an inspiring conference with an ambitious manifesto, ultimately aiming to improve patient care. Unfortunately, we are still failing to reach the majority of the medical community. To do this, we need to learn from other social movements to understand their roadmaps and how to improve ours. This will enable movements like Evidence Live to be successfully implemented into practice.

\section{MISREPRESENTED OR UNVERIFIED AFFILIATIONS IN THE LITERATURE SHOULD BE ADDRESSED BY A CONSENSUS ON THE DEFINITION OF AFFILIATION}

Francisco Uribe, Vivienne Bachelet. Facultad de Ciencias Médicas, Universidad de Santiago de Chile, Santiago, Chile 